\title{
Exercise-Induced Myofibrillar Hypertrophy is a Contributory Cause of Gains in Muscle Strength
}

\author{
Christopher B. Taber ${ }^{1} \mathbb{D} \cdot$ Andrew Vigotsky $^{2} \cdot$ Greg Nuckols $^{3} \cdot$ Cody T. Haun ${ }^{4}$
}

Published online: 23 April 2019

C) Springer Nature Switzerland AG 2019

\section{Introduction}

The primary focus of this commentary is to discuss the relationship between training-induced increases in muscle size (i.e., hypertrophy) and changes in strength. Recently, Buckner et al. and Hornsby et al. debated the contribution of hypertrophy to strength and the role hypertrophy plays in sports performance; however, this is not a new discussion $[1,2]$. The exact contribution of hypertrophy to strength remains to be determined; yet, we feel certain considerations can provide clarity for future work. To provide these considerations, we begin by operationally defining both hypertrophy and strength. Thereafter, we address the strengthhypertrophy relationship through: (1) epistemological and statistical considerations, (2) molecular, mechanical, and single-fiber bases, and (3) exemplary training studies.

\section{Defining Hypertrophy and Strength}

Skeletal muscle hypertrophy has been described in the literature as an increase in muscle size. Muscle size can be described by its mass and/or volume. However, many measurement approaches are gross in nature, meaning they

This Commentary has a reply available at https://doi.org/10.1007/ s40279-019-01106-9.

Christopher B. Taber

taberc@sacredheart.edu

1 Department of Physical Therapy and Human Movement Science, Sacred Heart University, 5151 Park Avenue, Fairfield, CT, USA

2 Department of Biomedical Engineering, Northwestern University, Evanston, IL, USA

3 Department of Exercise and Sport Science, University of North Carolina at Chapel Hill, Chapel Hill, NC, USA

4 Department of Exercise Science, LaGrange College, LaGrange, GA, USA provide little insight into the constituents that contribute to an alteration in mass and volume (e.g., fluid and protein). That is, gross assessments of muscle hypertrophy do not provide a direct measurement of alterations in muscle protein abundance or fluid, both of which can influence muscle function (e.g., strength).

Since the underlying changes that affect muscle size can differentially affect muscle function, we contend that the term hypertrophy, as a biological construct, be carefully employed, especially when a causal relationship is considered. Furthermore, as in other tissues, we posit that different types of hypertrophy can occur, which can differentially affect function and, consequently, the association between hypertrophy and strength [3, 4]. Finally, we contend that the measurement used to assess changes in muscle size can affect the statistical relationship, or lack thereof, with strength outcomes.

Therefore, for the purpose of this commentary, we carefully posit the following definition of skeletal muscle hypertrophy: An increase in muscle size accompanied by an increase in myofibrillar protein. However, we feel it more appropriate for research to decipher various types of hypertrophy that may occur in concert or as distinct responses to specific training protocols: (1) connective tissue $[5,6]$, (2) myofibrillar [7, 8], and (3) sarcoplasmic [8, 9]. Since each fraction of muscle tissue has been shown to be responsive to exercise training, with only myofibrillar hypertrophy intuited to contribute directly to force production capacity, associations between hypertrophy and strength should be made cautiously. Stated differently, without a direct assessment of myofibrillar protein alterations, casual relationships between hypertrophy and increases in strength are suspect.

Although strength is somewhat easier to define and measure given its mechanical nature, how it is measured and associated with hypertrophy is also a source of variance. Strength is a multifaceted skill and can be defined as the ability to produce force against an external resistance [10, 11]. Strength can be expressed on a spectrum (e.g., 0-100\%) and can be measured in multiple ways (e.g., isometric, dynamic, voluntary, involuntary). The strength construct being assessed further influences 
the observed association with hypertrophy. Considering this, an epistemological and statistical discussion is warranted.

\section{Epistemological and Statistical Considerations}

The word relationship is ontologically vague. Given that this piece is predicated on relationship being well defined, it is necessary to provide context via an operational definition. Relationships may be broadly thought of as causal or purely associational. While there are subcategories of each of these, we are most interested in hypertrophy having a causal role in strength gain. Still, the word causal can be ambiguous, as there exist multiple types of causal relationships: (1) necessary (if not A, then not B), (2) sufficient (if A then B), and (3) contributory (neither necessary nor sufficient).

We contend that myofibrillar hypertrophy has a causal impact on gains in muscular strength, but that it is a contributory causal relationship. We concede that strength can increase without increases in muscle size (not necessary) [12], and that an increase in muscle size may not be accompanied by an increase in strength (not sufficient) [13], but that does not negate the possibility that hypertrophy is

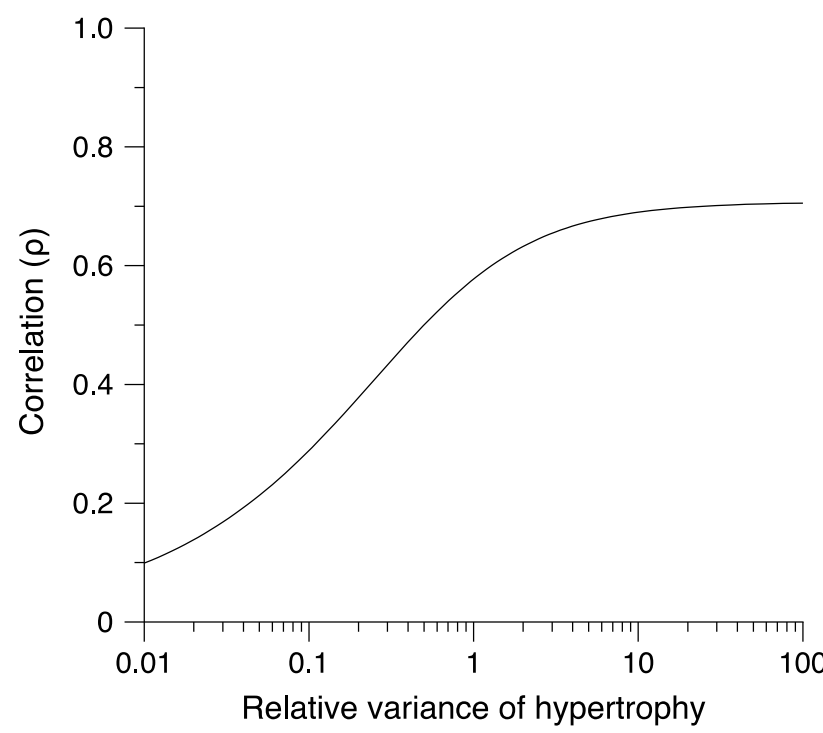

Fig. 1 The dependence of correlative strength on the relative weight of muscle size to other factors that influence strength. Consider a model where strength $=$ muscle size $\times$ all other factors that influence strength (neural factors, technique, mechanical factors, etc.). Within this model, we assume that the other factors play a larger $(<1$, above) or smaller $(>1$, above) role than hypertrophy, that there is variability in both the hypertrophy response to training, and the response of the other factors influencing strength, and that within each individual, hypertrophic adaptations and "other" adaptations are independent of each other (i.e., large hypertrophy adaptations do not guarantee large neural adaptations, and vice versa). Given these assumptions, there could be a very weak between-subject correlation between hypertrophy and strength gains; however, it is clear from the model that hypertrophy has a direct, causal impact on strength gains still a contributory cause of strength increases. Indeed, the contribution of muscle size to strength, relative to all other factors that influence strength, will undoubtedly affect the observed correlation (Fig. 1). Thus, without measuring and statistically modeling every variable that could contribute to strength, it can be argued that any correlation between hypertrophy and strength gain is confounded. As such, drawing casual conclusions, in either direction, from a single line of evidence is likely specious; multiple lines of evidence, combined with theory, must be considered.

We posit that substantial confounding due to "other factors" is likely prevalent for relatively untrained lifters. For lifters with more training experience, we posit that fewer adaptations are taking place among those "other" factors, thus increasing the correlation between hypertrophy and strength gains. We, therefore, find the question of "Does hypertrophy contribute to strength gain?" less interesting than "To what extent and under what circumstances does hypertrophy contribute to strength gain?"

\section{Molecular, Mechanical, and Single-Fiber Basis of Relationship}

Active muscular force production is primarily the result of contractile protein interactions at the sarcomere level. Upon neural recruitment, calcium release, and actin exposure, a specific amount of force is produced by myosinactin interaction. This force is transduced laterally and longitudinally in a healthy myofibril $[14,15]$. Longitudinally, the sum of these forces and transduction through a tendon to a bone produces skeletal movement. Laterally, forces are transmitted to connective tissue, which may change how forces in series and in parallel are combined [16, 17].

Since the site of active force generation in a myofibril is the interaction of myosin and actin, an increase in myosin and actin filaments in parallel can increase the capacity of a myofibril to produce force. This is one factor contributing to increases in whole muscle force, along with neural and connective tissue adaptations.

To revisit a principal historical finding in muscle physiology, Huxley's work showed us that the amount of force produced by a sarcomere during isometric contraction can be represented as the number of strongly-bound cross-bridges [18]. Conceptually, more sarcomeres in a myofibril through training-induced hypertrophy (e.g., sarcomerogenesis resulting in more sarcomeres in parallel) increases the force production capacity of a myofibril [19]. This is grounded in the basic mechanical tenet that forces in parallel add. For this reason, myofibrillar hypertrophy should directly increase strength.

A direct quotation from Miller et al.'s 2014 synthesis of literature on myofilament adaptations is appropriate here: 
"....at the fiber level, the amount of isometric force produced is equal to the total number of heads interacting in each half-sarcomere (each half-sarcomere must produce identical forces or the sarcomere will change its length). Thus, removal of myosin heads, either from the ends of the thick filament or randomly throughout the thick filament, or a reduction in the number of thin filaments would reduce the number of heads able to interact in a half-sarcomere and result in lower force production. Although fiber CSA is commonly measured in healthy adults as well as during aging, disuse, and disease, changes in myofilament protein content and ultrastructure have not been routinely examined, especially in combination with contractile measurements [20]" p. 3.

Therefore, the supposed disconnect between hypertrophy and strength in various studies could be due to: (1) hypertrophy not being myofibril-driven, (2) myofibrillar growth unaccompanied by complementary adaptations to other pertinent tissues or the muscle fiber itself required to express the new capacity, or (3) an examination of strength before the contribution of hypertrophy can be expressed. Indeed, it is plausible that a now larger muscle requires time to learn how to use (i.e., delayed training effect). Larger isolated single muscle fibers tend to produce more force than smaller fibers upon stimulation [21]. Collectively, investigations tend to show a maintenance of or increase in specific tension $\left(\mathrm{N} / \mu \mathrm{m}^{2}\right)$ after resistance training (RT)-induced hypertrophy [22-24]. Thus, the relationship of hypertrophy to strength is ancillary, but the methods used to identify this have often squandered the opportunity to do so.

\section{Training Studies}

Based on the prior physiological underpinnings of muscle mechanics and measurement techniques, we can examine studies that measure hypertrophy and changes in muscle strength. It is important to consider the population, training status, and measurement techniques when investigating the relationship between hypertrophy and strength gain. Because the contributions to strength outcomes are multifactorial and may vary with training age, a critical analysis of the literature should be performed.

Strength can be developed through a variety of RT methods and may be mediated by neural factors, skill acquisition, mechanical changes, and morphological alterations. It has been proposed that early changes in strength are primarily driven by neural factors and skill acquisition, and later changes are primarily mediated by muscular hypertrophy $[25,26]$. If that is true, then one would anticipate the association between hypertrophy and strength gains to increase as training age increases. Because the training process is a "long-term investment," it is necessary to understand when particular physiological mechanisms may play a larger role compared to others in the improvement of strength.

When examining short-term studies where subjects are naïve to $\mathrm{RT}$, relative increases in strength outpace relative increases in muscle size. This is readily observable in a multiple studies where strength increases in the first 4-6 weeks with little-to-no appreciable hypertrophy [27, 28], and is supported by several studies that examined strength changes resulting from short-term training interventions (12-24 weeks). In these studies, the correlations between muscle hypertrophy and strength changes are low, with hypertrophy accounting for as little as $2-28 \%$ of the variance in strength improvement [13, 29-32]. Contrasting these findings are studies with homogeneous groups of trained individuals ( $>1$ year RT), which suggest that, as training status increases, hypertrophy accounts for a greater percentage of the variance in strength gain [29, 33].

As opposed to short-term studies in untrained individuals, longer-term studies on trained individuals suggest that hypertrophy-as assessed via changes in body mass and fiber cross-sectional area-account for $\sim 65 \%$ of the variance in strength gain $[34,35]$. Such findings in trained individuals are further supported by the work of Appleby and colleagues, who evaluated professional rugby players over 2 years of RT [36]. Investigators found that squat strength increases were strongly correlated with relative changes in lean mass index $\left(R^{2}=44-77 \%\right)$ [36].

The findings of the aforementioned studies in trained individuals are corroborated by cross-sectional studies with elite athletes, which suggest that muscle size accounts for an even larger percentage of variance in strength $\left(R^{2} \geq 70 \%\right)$ [37-41]. While these data do not necessarily suggest that changes in muscle size are related to changes in strength, they do still indicate that athletes with more muscle mass in relation to their height have a competitive advantage in the sports of powerlifting and Olympic weightlifting. The tightness of these associations in light of the mechanistic theory, we believe, suggests that hypertrophy provides lifters with a competitive advantage.

Finally, while the studies presented hitherto address the question of whether those who gain more muscle also get stronger (between-subject), it has been suggested that the question of greater interest is if an individual will get stronger as they gain more muscle (within-subject) [42]. Indeed, such analyses explain a much greater percentage of variance in strength gain than do between-subject analyses $[42,43]$. While this work remains in its infancy and has room for improvement, both methodologically and statistically, we believe the early results to be both intriguing 
and thought-provoking in helping to elucidate the strengthhypertrophy relationship.

\section{Conclusions}

We have presented theoretical and longitudinal evidence that strength acquisition in the long term is enhanced by hypertrophy. We have provided evidence that mechanical and molecular factors support the hypothesis that hypertrophy enhances strength. In doing so, we have illuminated issues with different methods and their respective abilities to elucidate relationships between strength and hypertrophy. Finally, we have provided evidence that short-term studies utilizing untrained subjects may not fully capture the influence of hypertrophy on strength. We appreciate the multitude of factors that may play a role in the acquisition of strength; we conclude that in the long-term muscular hypertrophy contributes to strength.

\section{Reply to Loenneke et al.}

We appreciate the opportunity to respond to Loenneke et al. [44] and for these authors' willingness to exchange points on this matter. As we noted in our initial writing, evidence supports the contention that hypertrophy is neither a necessary nor sufficient cause of improved strength in all contexts. However, we feel evidence overwhelmingly supports hypertrophy as a contributory cause of improvements in strength.

Loenneke et al. argue that associational evidence used to support the theory that hypertrophy is related to changes in strength is simply "correlating error/random biological variability". However, from a statistical standpoint, we contend that this point is not supported. By definition, on average, random error should have zero correlation with other random error, assuming that the random error is independent. In the case of assessing muscle size and strength, it is unclear why covariance would be present between strength and hypertrophy measurement/biological variability, and the authors have not supported this underlying premise. Indeed, mathematical proofs show that, on average, measurement error decreases correlation [45], which suggests that the correlations we observe in the literature are weaker than what is present in nature.

Loenneke et al. provide evidence that strength gains can occur without accompanying hypertrophy, and that similar hypertrophy does not guarantee similar strength gains. However, that evidence is not inconsistent with the contention that hypertrophy has a contributory causal effect on strength gains, as a contributory cause is one that is neither necessary nor sufficient. Indeed, in a training study, one mechanism can increase while another decreases, making the role of a single mechanism difficult to untangle. Since an experiment that assesses all potential mechanisms would be nearly impossible to complete in humans, multiple lines of work may be needed to establish or refute hypertrophy as a contributory cause. That is, the question may not be able to be answered through a single experiment, but rather, many experiments combined with modeling may be needed [42].

Addressing Loenneke et al.'s point concerning low-load versus high-load training, they state correctly that strength increases more with high-load training in the movements being trained. However, a recent meta-analysis [46] found that high-load training produced larger gains in dynamic strength, while also finding no significant differences between high-load and low-load training in both hypertrophy and isometric strength gains. This suggests that hypertrophy increases the muscle's general ability to produce force, regardless of loading zone, and that high-load training additionally leads to superior skill acquisition for dynamic strength testing.

Finally, the impact of non-training stimuli on hypertrophy and strength cannot be overlooked. Bhasin et al. found that supraphysiological doses of testosterone ( $600 \mathrm{mg} / \mathrm{week})$ caused both hypertrophy (15.7\% increase in thigh muscle volume) and strength gains (17.7\% increase in leg press strength) without any RT stimulus [47]. The lack of training stimulus and the similarity between hypertrophy and strength gains suggest that the strength gains were driven by muscle hypertrophy. Considering these points, it seems evident that hypertrophy can be considered a contributory cause of improvements in strength.

\section{Compliance with Ethical Standards}

Funding No financial support was received for the preparation of this manuscript.

Conflict of interest Cody Haun, Christopher Taber, Andrew Vigotsky, and Greg Nuckols declare that they have no conflict of interest.

\section{References}

1. Buckner SL, Dankel SJ, Mattocks KT, Jessee MB, Mouser JG, Counts BR, et al. The problem of muscle hypertrophy: revisited. Muscle Nerve. 2016;54(6):1012-4.

2. Hornsby WG, Gentles JA, Haff GG, Stone MH, Buckner SL, Dankel SJ, et al. What is the impact of muscle hypertrophy on strength and sport performance? J Strength Cond Res. 2018;40(6):99-111.

3. Mihl C, Dassen W, Kuipers H. Cardiac remodelling: concentric versus eccentric hypertrophy in strength and endurance athletes. Neth Heart J. 2008;16(4):129-33.

4. Johansson B. Different types of smooth muscle hypertrophy. Hypertension. 1984;6 Pt 6(2):III64.

5. Stone MH. Implications for connective tissue and bone alterations resulting from resistance exercise training. Med Sci Sports Exerc. 1988;20(5 Suppl):S162-8. 
6. Kjaer M. Role of extracellular matrix in adaptation of tendon and skeletal muscle to mechanical loading. Physiol Rev. 2004;84(2):649-98.

7. Cribb PJ, Hayes A. Effects of supplement-timing and resistance exercise on skeletal muscle hypertrophy. Med Sci Sports Exerc. 2006;38(11):1918-25.

8. Roberts M, Romero M, Mobley C, Mumford P, Roberson P, Haun C, et al. Skeletal muscle mitochondrial volume and myozenin-1 protein differences exist between high versus low anabolic responders to resistance training: PeerJ Preprints2018. Report no.: 2167-9843.

9. MacDougall J, Sale D, Elder G, Sutton J. Muscle ultrastructural characteristics of elite powerlifters and bodybuilders. Eur J Appl Physiol Occup Physiol. 1982;48(1):117-26.

10. Siff M. Biomechanical foundations of strength and power training. London: Blackwell Scientific Ltd; 2000. p. 103-39.

11. Stone MH. Position statement: explosive exercise and training. Strength Cond J. 1993;15(3):7-15.

12. Parente V, D'Antona G, Adami R, Miotti D, Capodaglio P, De Vito $\mathrm{G}$, et al. Long-term resistance training improves force and unloaded shortening velocity of single muscle fibres of elderly women. Eur J Appl Physiol. 2008;104(5):885.

13. Ahtiainen JP, Walker S, Peltonen H, Holviala J, Sillanpää E, Karavirta $\mathrm{L}$, et al. Heterogeneity in resistance training-induced muscle strength and mass responses in men and women of different ages. Age. 2016;38(1):10.

14. Street SF. Lateral transmission of tension in frog myofibers: a myofibrillar network and transverse cytoskeletal connections are possible transmitters. J Cell Physiol. 1983;114(3):346-64.

15. Ramaswamy KS, Palmer ML, Van Der Meulen JH, Renoux A, Kostrominova TY, Michele DE, et al. Lateral transmission of force is impaired in skeletal muscles of dystrophic mice and very old rats. J Physiol. 2011;589(5):1195-208.

16. Huijing PA. Muscle as a collagen fiber reinforced composite: a review of force transmission in muscle and whole limb. J Biomech. 1999;32(4):329-45.

17. Jones D, Rutherford O, Parker D. Physiological changes in skeletal muscle as a result of strength training. Q J Exp Physiol. 1989;74(3):233-56.

18. Huxley AF. Muscle structure and theories of contraction. Prog Biophys Biophys Chem. 1957;7:255-318.

19. van der Pijl R, Strom J, Conijn S, Lindqvist J, Labeit S, Granzier H, et al. Titin-based mechanosensing modulates muscle hypertrophy. J Cachexia Sarcopenia Muscle. 2018;9(5):947-61.

20. Miller MS, Callahan DM, Toth MJ. Skeletal muscle myofilament adaptations to aging, disease, and disuse and their effects on whole muscle performance in older adult humans. Front Physiol. 2014;5:369.

21. Gilliver S, Degens H, Rittweger J, Sargeant A, Jones D. Variation in the determinants of power of chemically skinned human muscle fibres. Exp Physiol. 2009;94(10):1070-8.

22. Trappe S, Williamson D, Godard M, Porter D, Rowden G, Costill D. Effect of resistance training on single muscle fiber contractile function in older men. J Appl Physiol. 2000;89(1):143-52.

23. Widrick JJ, Stelzer JE, Shoepe TC, Garner DP. Functional properties of human muscle fibers after short-term resistance exercise training. Am J Physiol Regul Integr Comp Physiol. 2002;283(2):R408-16.

24. Dankel SJ, Kang M, Abe T, Loenneke JP. Resistance training induced changes in strength and specific force at the fiber and whole muscle level: a meta-analysis. Eur J Appl Physiol. 2019;119(1):265-78

25. Sale DG. Neural adaptation to resistance training. Med Sci Sports Exerc. 1988;20(5 Suppl):S135-45.

26. Gabriel DA, Kamen G, Frost G. Neural adaptations to resistive exercise. Sports Med. 2006;36(2):133-49.

27. Moritani T. Neural factors versus hypertrophy in the time course of muscle strength gain. Am J Phys Med. 1979;58(3):115-30.
28. Seynnes OR, de Boer M, Narici MV. Early skeletal muscle hypertrophy and architectural changes in response to high-intensity resistance training. J Appl Physiol. 2007;102(1):368-73.

29. Balshaw TG, Massey GJ, Maden-Wilkinson TM, Lanza MB, Folland JP. Neural adaptations after 4 years vs 12 weeks of resistance training vs untrained. Scand J Med Sci. 2019;29:348-59.

30. Maeo S, Shan X, Otsuka S, Kanehisa H, Kawakami Y. Neuromuscular adaptations to work-matched maximal eccentric versus concentric training. Med Sci Sports Exerc. 2018;50(8):1629.

31. Erskine RM, Jones DA, Maffulli N, Williams AG, Stewart CE, Degens $H$. What causes in vivo muscle specific tension to increase following resistance training? Exp Physiol. 2011;96(2):145-55.

32. Erskine RM, Fletcher G, Folland JP. The contribution of muscle hypertrophy to strength changes following resistance training. Eur J Appl Physiol. 2014;114(6):1239-49.

33. Narici MV, Hoppeler H, Kayser B, Landoni L, Claassen H, Gavardi $\mathrm{C}$, et al. Human quadriceps cross-sectional area, torque and neural activation during 6 months strength training. Acta Physiol Scand. 1996;157(2):175-86.

34. Cribb PJ, Williams AD, Stathis CG, Carey MF, Hayes A. Effects of whey isolate, creatine and resistance training on muscle hypertrophy. Med Sci Sports Exerc. 2007;39(2):298-307.

35. Baker D, Wilson G, Carlyon R. Periodization: the effect on strength of manipulating volume and intensity. J Strength Cond Res. 1994;8(4):235-42.

36. Appleby B, Newton RU, Cormie P. Changes in strength over a 2-year period in professional rugby union players. J Strength Cond Res. 2012;26(9):2538-46.

37. Siahkouhian M, Hedayatneja M. Correlations of anthropometric and body composition variables with the performance of young elite weightlifters. J Hum Kinet. 2010;25:125-31.

38. Blazevich AJ, Coleman DR, Horne S, Cannavan D. Anatomical predictors of maximum isometric and concentric knee extensor moment. Eur J Appl Physiol. 2009;105(6):869-78.

39. Trezise J, Collier N, Blazevich AJ. Anatomical and neuromuscular variables strongly predict maximum knee extension torque in healthy men. Eur J Appl Physiol. 2016;116(6):1159-77.

40. Lietzke M. Relation between weight-lifting totals and body weight. Science. 1956;124(3220):486-7.

41. Brechue WF, Abe T. The role of FFM accumulation and skeletal muscle architecture in powerlifting performance. Eur J Appl Physiol. 2002;86(4):327-36.

42. Vigotsky AD, Schoenfeld BJ, Than C, Brown JM. Methods matter: the relationship between strength and hypertrophy depends on methods of measurement and analysis. PeerJ. 2018;6:e5071.

43. Loenneke JP, Rossow LM, Fahs CA, Thiebaud RS, Grant Mouser J, Bemben MG. Time-course of muscle growth, and its relationship with muscle strength in both young and older women. Geriatr Gerontol Int. 2017;17(11):2000-7.

44. Loenneke JP, Buckner SL, Dankel SJ, Abe T. Exercise-induced changes in muscle size do not contribute to exercise-induced changes in muscle strength. Sports Med. 2019. https://doi.org/10.1007/s4027 9-019-01106-9.

45. Fuller WA. Measurement error models, vol. 305. Hoboken: Wiley; 2009.

46. Schoenfeld BJ, Grgic J, Ogborn D, Krieger JW. Strength and hypertrophy adaptations between low- vs. high-load resistance training: a systematic review and meta-analysis. J Strength Cond Res. 2017;31(12):3508-23.

47. Bhasin S, Woodhouse L, Casaburi R, Singh AB, Bhasin $\mathrm{D}$, Berman $\mathrm{N}$, et al. Testosterone dose-response relationships in healthy young men. Am J Physiol Endocrinol Metab. 2001;281(6):E1172-81. 